\title{
Propiedades psicométricas del TEOSQ adaptado a la educación física en estudiantes ecuatorianos
}

\section{Evelia Franco $^{1}$ (i), Javier Coterón ${ }^{2}$ (D), Jorge Brito ${ }^{3}$}

${ }^{1}$ Centro Universitario Cardenal Cisneros, Departamento de Didácticas Específicas, Universidad de Alcalá, Madrid, España.

${ }^{2}$ Facultad de Ciencias de la Actividad Física y del Deporte, Universidad Politécnica de Madrid, Madrid, España.

${ }^{3}$ Facultad de Filosofía, Universidad de Cuenca, Cuenca, Ecuador.

Autor para correspondencia: evelia.franco@cardenalcisneros.es

Fecha de recepción: 27 de septiembre 2016 - Fecha de aceptación: 23 de noviembre 2016

\section{RESUMEN}

El propósito de este trabajo fue analizar las propiedades psicométricas de la versión española del Cuestionario de Orientación al Ego y a la Tarea (TEOSQ) adaptada a la EF ${ }^{1}$, en un contexto ecuatoriano. En el estudio participaron 580 estudiantes de EF con edades comprendidas entre 12 y 16 años $(M=$ 13.15; $D T=1.29$ ). Los resultados de los análisis factoriales apoyaron la estructura del instrumento obtenida en estudios previos. La herramienta mostró un nivel adecuado de consistencia interna $\left(\alpha_{E g o}=\right.$ $\left..86 \mathrm{y} \alpha_{\text {Tarea }}=.80\right)$ y las diferencias de género indicaron que los chicos mostraron mayor orientación al ego que las chicas en línea con lo encontrado en la literatura. Los resultados permiten considerar la versión adaptada a la EF del TEOSQ en castellano como un instrumento fiable y válido para valorar la orientación de los estudiantes hacia la tarea y hacia el ego en el contexto ecuatoriano.

Palabras clave: TEOSQ, orientación motivacional, propiedades psicométricas, estudiantes ecuatorianos.

\begin{abstract}
The objective of the study was to analyze the psychometric properties of the Spanish version of the Task and Ego Orientation Sport Questionnaire (TEOSQ) adapted to $\mathrm{PE}^{2}$, in an Ecuadorian context. Five hundred and eighty PE students aged 12 to $16(M=13.15 ; S D=1.29)$ participated in the study. Factor analysis results confirmed the structure of the instrument obtained in previous studies. The questionnaire showed an acceptable internal consistency $\left(\alpha_{E g o}=.86 \mathrm{y} \alpha_{T a s k}=.80\right)$. The differences per gender indicated that boys show greater ego orientation than girls in line with existing literature. Results allow considering the Spanish version of TEOSQ adapted to PE a reliable and valid instrument to measure motivational orientation of students in an Ecuadorian context.
\end{abstract}

Keywords: TEOSQ, motivational orientation, psychometric properties, Ecuadorian students.

\section{INTRODUCCIÓN}

En los últimos años existe un creciente interés en el análisis de los factores relacionados con la motivación en contextos de actividad físico-deportiva (Moreno-Murcia et al., 2012; Moreno-Murcia et

\footnotetext{
1 EF: Educación Física

2 PE: Physical Education
} 
al., 2013) debido a la influencia que dichos factores ejercen sobre la adherencia a la práctica de actividad física la cual conlleva innegables beneficios sobre la salud física y psicosocial cuando se realiza de forma regular. Las clases de educación física, como forma de contacto cotidiano de niños y adolescentes con la actividad física, deberían ayudar a la promoción y el fomento de la actividad física entre el alumnado, siendo este uno de sus principales objetivos.

Una perspectiva desde la que se ha abordado en las últimas décadas el estudio de la motivación en educación física es la teoría de las metas de logro (Nicholls, 1989). Esta teoría plantea que las personas son organismos intencionales dirigidos por unos objetivos que consisten en esforzarse por demostrar competencia o habilidad en contextos de logro y que, dependiendo del concepto de habilidad que tengan, van a presentar una implicación a la tarea o al ego. Este estado de implicación dependerá de unos factores contextuales (clima motivacional) y disposicionales (orientación motivacional). Una orientación a la tarea implica una preocupación por el desarrollo personal, el éxito se define en términos de mejora y la percepción de capacidad es autorreferenciada. Por el contrario, una orientación al ego implica la utilización de información normativa para definir el éxito y decidir la capacidad que se tiene en la realización de actividades concretas.

En el marco de estos postulados se construyó el Cuestionario de Orientación al Ego y a la Tarea en el Deporte (Duda, 1989), una de las herramientas más utilizadas para la evaluación de las orientaciones motivacionales en contextos deportivos (e.g., Baric et al., 2014; Stuntz \& Weiss, 2012). La versión inicial consta de 13 ítems agrupados en dos factores: orientación a la tarea, medida con siete ítems; y orientación al ego, medida con los seis ítems restantes. El encabezamiento del cuestionario es "Yo me siento con más éxito en el deporte cuando...", y se responden mediante una escala tipo Likert que va de1 (muy en desacuerdo) a 5 (muy de acuerdo).

Walling \& Duda (1995) desarrollaron una versión del TEOSQ adaptada a la EF escolar conformada por 16 ítems cambiando el enunciado inicial por "Yo siento que tengo más éxito en las clases de EF cuando...". Las investigaciones en este ámbito (Todorovich \& Curtner-Smith, 2002) han puesto de manifiesto la validez y fiabilidad del TEOSQ adaptado a la EF, siendo considerado un instrumento muy consistente y útil para medir las diferencias individuales en las perspectivas de meta en este contexto. En el contexto español Peiró \& Sanchís (2004) realizaron una traducción al español de la versión del TEOSQ de 16 ítems adaptada a la EF. En este estudio, en el que participaron 774 estudiantes de ESO, se comprobó que las propiedades psicométricas del instrumento eran adecuadas para ser utilizadas en un contexto de EF en España.

Tal y como señalan Duda y colaboradores (Duda \& Allison, 1990; Duda \& Hayashi, 1998) la investigación en el ámbito de la psicología del deporte y de la actividad física requiere la incorporación de análisis interculturales con el fin de evitar la propagación de teorías que puedan no ser generalizables, ante la posible existencia de factores ligados a la cultura o la etnia que afecten a la variabilidad en los conocimientos, emociones y comportamientos abordados desde las teorías psicológicas del deporte y el ejercicio. En esta línea, resulta indispensable la validación de instrumentos susceptibles de ser utilizados en diferentes contextos. El TEOSQ en el ámbito deportivo ha sido traducido a diversos idiomas y contextos culturales (Cindi \& Koenraad, 2005; De Andrade et al., 2008; Fonseca \& Brito, 2005; Guivernau \& Duda, 1994; Kim et al., 2003; Li et al., 1996; López-Walle et al., 2011; Van-Yperen \& Duda, 1999; Wakayama et al., 2002).

Como hemos señalado anteriormente, el TEOSQ presenta unas propiedades psicométricas adecuadas entre estudiantes de EF españoles (Peiró \& Sanchis, 2004). Aunque Ecuador y España comparten una misma lengua materna, ambos países pertenecen a contextos culturales diferentes que suponen unas características idiosincráticas diferenciadas. La validez transcultural de los instrumentos no puede, por tanto, presumirse sin más en contextos culturales que compartan un substrato lingüístico común (Hambleton, 2006; Muñiz et al., 2013). Considerando la necesidad de comprobar la equivalencia en la medida de los diferentes grupos culturales con el fin de llevar a cabo estudios transculturales, el propósito de este estudio es evaluar las propiedades psicométricas del TEOSQ en una muestra de estudiantes de EF ecuatorianos utilizando el análisis factorial confirmatorio para contrastar la aplicabilidad de la estructura obtenida utilizando una muestra de España. 


\section{MATERIALES Y MÉTODOS}

\subsection{Participantes}

La muestra está formada por 588 estudiantes de educación física de la ciudad de Cuenca (Ecuador), de los cuales 406 eran chicos y 174 chicas con edades comprendidas entre los 12 y los 16 años $(M=13.15$; $D T=1.29$ ).

\subsection{Instrumento}

Se utilizó la versión española (Peiró \& Sanchis, 2004) del Cuestionario de Orientación al Ego y a la Tarea en el Deporte (TEOSQ; Duda, 1989) adaptado a la EF (Walling \& Duda, 1995). En las instrucciones se pide a los estudiantes que piensen cuándo se sienten con más éxito en la clase de EF mediante la pregunta "Yo siento que tengo más éxito en las clases de educación física cuando...". El TEOSQ consta de 16 ítems que evalúan las metas de logro a través de sus dos dimensiones reflejadas en 8 ítems cada una; la orientación a la tarea (p.e., "cuando soy capaz de esforzarme en la práctica de una habilidad") y la orientación al ego (e.g., "cuando lo puedo hacer mejor que mis compañeros"). Las respuestas se recogen mediante una escala tipo Likert de cinco puntos que oscila de 1 (muy en desacuerdo) a 5 (totalmente de acuerdo).

\subsection{Procedimiento}

\section{Estudio preliminar}

El estudio preliminar incluyó dos pasos: una revisión lingüística y cultural por expertos de la educación física que conocían en profundidad el español utilizado en ambos contextos; y un estudio piloto cualitativo en una pequeña muestra de estudiantes. Se han considerado las principales directrices elaboradas para la adaptación de los test (Hambleton, 2006). Como segundo paso, se aplicó a 30 estudiantes para evaluar si el instrumento era claro y adecuado.

\section{Estudio principal}

Se estableció contacto con los equipos directivos y/o responsables del departamento de EF de los diferentes centros de enseñanza para informarles de los objetivos del estudio y pedirles su colaboración. Se solicitaron y obtuvieron las autorizaciones pertinentes para que los alumnos pudieran participar en el estudio. Tras la obtención de los permisos pertinentes conforme las indicaciones de los principios éticos y códigos de conducta de la American Psychological Association (2002), la administración de los cuestionarios fue llevada a cabo por el investigador principal y un equipo previamente formado entre marzo y diciembre de 2014. Los cuestionarios se administraron durante una clase de EF en ausencia del profesor, dando las instrucciones necesarias para la cumplimentación e insistiendo en el anonimato y sinceridad en las respuestas. La participación fue voluntaria y el tiempo requerido para rellenar los cuestionarios fue de aproximadamente 25 minutos, variando ligeramente según la edad de los estudiantes.

\subsection{Análisis de los datos}

Se analizó la consistencia interna mediante el coeficiente alfa $(\alpha)$ (Cronbach, 1951). Se analizaron los ítems utilizando como criterio que la correlación del ítem con la escala fuera superior a .30 excluido el propio ítem, que el coeficiente alfa no aumentase si se eliminara ese ítem y que tuvieran suficiente poder discriminativo. Se analizó la estructura factorial a través de un análisis factorial exploratorio y confirmatorio. En último lugar, para analizar las evidencias de validez externa, se realizaron pruebas $t$ para examinar las diferencias por género en las dos orientaciones. Los datos fueron analizados utilizando los paquetes estadísticos SPSS 20.0 y Amos 20.0. 


\section{RESULTADOS}

\subsection{Estructura factorial exploratoria}

La prueba de Bartlett sobre la matriz de correlaciones entre los ítems $($ Bartlett $=2885.77, \mathrm{gl}=120$, $p<.001)$ mostró que las variables del TEOSQ no eran independientes. La medida de adecuación de Kaiser-Meyer-Olkin mostró un valor significativo $(K M O=.89)$ indicando que las correlaciones entre pares de variables pueden ser medianamente explicadas por las restantes variables del instrumento (Kaiser, 1974).

Un análisis de componentes principales con rotación varimax, dio como resultado dos componentes con valor propio mayor que la unidad. El factor 1 obtuvo un valor propio de 4.43, lo que representa un $27.68 \%$ de la varianza total. El factor 2 obtuvo un valor propio de 3.16, lo que representa un $19.77 \%$ de la varianza total. El porcentaje acumulado de los dos factores explica el $47.45 \%$ de la varianza. Utilizando .50 como criterio de saturación interpretable en la rotación ortogonal, los ítems 1 , $3,5,7,9,11,13$ y 15 , relativos a orientación a la tarea, saturan en el primer factor; y los ítems restantes, relativos a la orientación al ego, en el segundo factor.

\subsection{Consistencia interna y análisis de ítems}

El coeficiente alfa fue de .86 en la orientación al ego y de .80 en la orientación a la tarea. En ninguno de los dos factores la eliminación de algún ítem conseguiría aumentar la consistencia interna de la escala. Para analizar el poder discriminativo de los ítems se calculó la diferencia entre las medias de las puntuaciones obtenidas en cada uno de los ítems entre los participantes del primer cuartil y del último cuartil. Todas las diferencias resultaron significativas mostrando todos los ítems poder discriminativo.

\subsection{Análisis factorial confirmatorio}

En la Figura 1 se muestra el modelo testado en el análisis factorial confirmatorio. El coeficiente de curtosis de Mardia fue de 364.9, lo cual indicó la no normalidad de los datos; para este modelo la normalidad fue asumida para valores por debajo de 288 , según la fórmula $p(p+2)$, donde $p$ es el número

.18

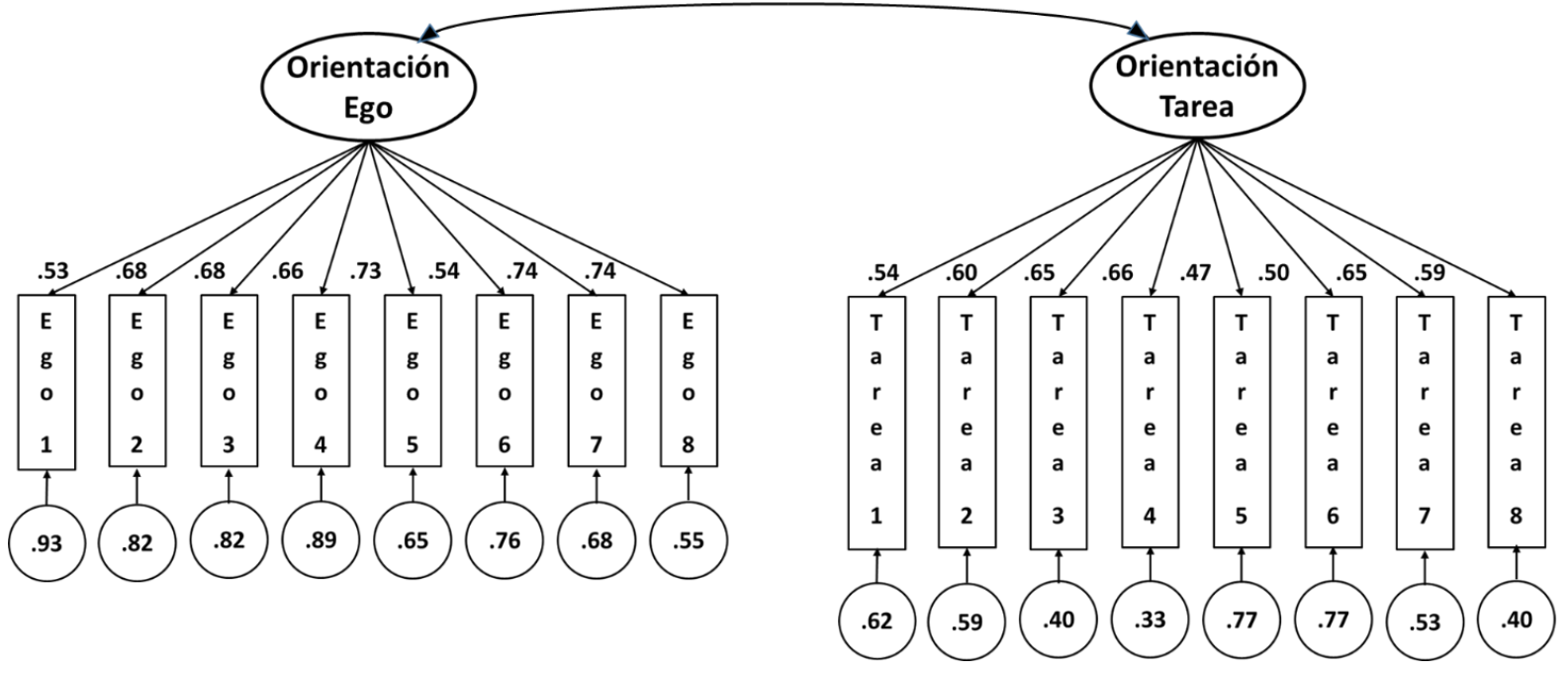

Figura 1. Análisis Factorial Confirmatorio del TEOSQ. Las elipses representan los factores y los rectángulos representan los diferentes ítems. Las varianzas residuales se muestran en los círculos pequeños. 
de variables observadas (Raykov \& Marcoulides, 2006). Se utilizó el método de estimación de máxima verosimilitud junto al procedimiento de bootstrapping, lo que permitió asumir que los datos eran robustos ante la falta de normalidad (Byrne, 2001).

Con el objetivo de comprobar la validez del modelo de medición, se consideraron diferentes índices de bondad de ajuste: chi-cuadrado, ratio entre chi-cuadrado y grados de libertad $\left(\chi^{2} / g l\right)$, RMSEA (Root Mean Square Error of Approximation), SRMR (Standardized Root Mean Square Residual) y los índices incrementales CFI (Comparative Fit Index) y TLI (Tucker-Lewis Index). Estos índices de bondad de ajuste son considerados aceptables cuando $\chi^{2} / g l$ presenta valores inferiores a 5 (Bentler, 1989), el RMSEA muestra valores por debajo de 0.8 (Browne \& Cudeck, 1993), al igual que el SRMR (Hu \& Bentler, 1999); y los índices incrementales (CFI y TLI) están por encima de .09 (Hu \& Bentler, 1995; Marsh et al., 2004).

Los índices obtenidos fueron: $\chi^{2}(103 N=588)=294.26, p=.00, \chi^{2} / g l=2.86, C F I=.93, T L I=$ $.92, S R M R=.06, R M S E A=.06$, reflejando una adecuada bondad de ajuste.

\subsection{Diferencias de género}

Las diferencias por género en las dos subescalas ego y tarea se examinaron mediante pruebas $t$. Los análisis indican que existen diferencias significativas en el factor ego $(t=2.94 ; p<0.01)$ estando el grupo de los chicos $(M=3.38 ; D T=.86)$ por encima del grupo de las chicas $(M=3.17 ; D T=.80)$. La prueba $t$ no resultó significativa para el factor tarea $(t=.77 ; p=.44)$ no hallándose diferencias entre chicos $(M=4.23 ; D T=.58)$ y chicas $(M=4.19 ; D T=.60)$.

\section{DISCUSIÓN}

El objetivo del presente estudio fue el estudio de las propiedades psicométricas de la versión del TEOSQ adaptada a la EF en estudiantes ecuatorianos. Siguiendo a Helms (1992), se testaron diferentes tipos de equivalencia que se han puesto a prueba en estudios transculturales anteriores. Concretamente, se testó la equivalencia lingüística (que la redacción del instrumento tuviese el mismo significado para un grupo cultural diferente) y la equivalencia psicométrica (que la herramienta midiese el mismo constructo que mide en otros contextos culturales) ya que los sujetos de ambos países, a pesar de compartir lengua materna, pueden presentar diferencias que requieren estudios de validación culturales específicos. Los resultados obtenidos al estudiar las propiedades psicométricas de la versión española del TEOSQ adaptada a la EF en una muestra de estudiantes ecuatorianos presentan similitudes esenciales con los obtenidos en el TEOSQ adaptado a otros contextos culturales tanto en las versiones adecuadas a contextos deportivos (Balaguer et al., 1996; Baric \& Horga, 2006; Fonseca \& Brito, 2005; Kim et al., 2003; López-Walle et al., 2011) como educativos (Papaioannou \& Macdonald, 1993; Peiró \& Sanchis, 2004; Todorovich \& Curtner-Smith, 2002; Walling \& Duda, 1995).

Los coeficientes $\alpha$ presentan unos valores elevados tanto en el ego como en la tarea (.86 y .80 respectivamente) demostrando una consistencia interna aceptable. Todos los ítems mostraron una correlación alta con su factor correspondiente no pareciendo aconsejable la supresión de ninguno de ellos puesto que, con dicha eliminación, no aumentaba la fiabilidad. Estos resultados sugieren una fiabilidad adecuada del instrumento para ser utilizado con estudiantes ecuatorianos.

Por su parte, la estructura factorial y la distribución de ítems entre factores concuerda con la postulación del modelo teórico y con la encontrado en trabajos previos reflejando claramente la existencia de dos factores conformados por ocho ítems cada uno. Además, las saturaciones de cada uno de los ítems con sus respectivos factores son altas, especialmente en el caso del factor "orientación al ego" en línea con lo hallado en la validación de la versión española (Peiró \& Sanchis, 2004). El hecho de que el modelo de medida esté en línea con las hipótesis derivadas de la teoría y resulte consistente con las mediciones obtenidas en otros estudios nos permite asumir una adecuada validez de constructo.

Con el fin de evaluar la validez de criterio del cuestionario, y en línea con lo desarrollado en trabajos anteriores en los que se habían comprobado las propiedades psicométricas de este instrumento (Balaguer et al., 1996; López-Walle et al., 2011; Peiró \& Sanchis, 2004), se procedió a analizar las 
diferencias en ambas orientaciones entre chicos y chicas. Las investigaciones en el ámbito de la EF han indicado que los chicos están más orientados al ego que las chicas observándose entre los primeros una mayor tendencia a compararse con el rendimiento de otros para valorar su propio éxito (Bakirtzoglou \& Ioannou, 2011; Baric et al., 2014; Papaioannou \& Kouli, 1999). Los resultados de nuestro estudio confirman los datos obtenidos en estos trabajos previos, de tal manera que son los alumnos varones los que presentan los mayores niveles de orientación al ego.

Podemos concluir, por tanto, que los análisis psicométricos del presente trabajo junto a la similitud de los resultados obtenidos con las versiones previas del instrumento evaluados en otros contextos culturales y lingüísticos, permiten considerar la versión adaptada a la EF del TEOSQ en castellano como un instrumento fiable y válido para valorar la orientación de los estudiantes de EF hacia la tarea y hacia el ego en el contexto ecuatoriano.

Estudios futuros pueden profundizar en el conocimiento de la motivación de los estudiantes de EF de diversos contextos. Esto, a través de un análisis de similitudes entre los patrones motivacionales de estos jóvenes con el objeto de diseñar estrategias de intervención que en la práctica pudieran ser aplicables en diferentes contextos.

\section{BIBLIOGRAFÍA}

American Psychological Association, 2002. Ethical principles of psychologists and code of conduct. American Psychologist, 57, 1060-1073.

Bakirtzoglou, P., P. Ioannou, 2011. Goal orientations, motivational climate and dispositional flow in Greek secondary education students participating in physical education lesson: differences based on gender. Facta Universitatis, 9(3), 295-306.

Balaguer, I., I. Castillo, I. Tomás, 1996. Análisis de las propiedades psicométricas del Cuestionario de Orientación al Ego y a la Tarea en el Deporte (TEOSQ) en su traducción al castellano. Psicológica, 17, 71-81.

Baric, R., S. Horga, 2006. Psychometric Properties of the Croatian Version of Task and Ego Orientation in Sport Questionnaire (CTEOSQ). Kinesiology, 38, 135-140.

Baric, R., J. Vlasic, S. Cecic Erpic, 2014. Goal orientation and intrinsic motivation for physical education: Does perceived competence matter? Kinesiology, 46(1), 117-126.

Bentler, P.M., 1989. EQS structural equations program manual. Los Angeles, CA: BMDP Statistical Software.

Browne, M.W., R. Cudeck, 1993. Alternative ways of assessing model fit. In: Bollen, K.A., J.S. Long (Eds.), Testing Structural Equation Models (pp. 136-162). Beverly Hills, CA: Sage.

Byrne, B.M., 2001. Structural equation modeling with Amos: Basic concepts, applications, and programming. Mahwah, NJ: Erlbaum.

Cindi, H.P.S., J.L. Koenraad, 2005. Motivational orientations in youth sport participation: Using Achievement Goal Theory and Reversal Theory. Personality and Individual Differences, 38, 605-618.

Cronbach, L.J., 1951. Coefficient alpha and the internal structure of tests. Psychometrika, 16, 297-334.

De Andrade, A., A. Salguero, R. González-Boto, S. Márquez, 2008. The relationship of participation motivation to gual orientations and perceived physical ability in Brazilian swimmers. Psychologia, 51, 157-169.

Duda, J.L., 1989. Relationship between task and ego orientation and the perceived purpose of sport among high school athletes. Journal of Sport and Exercise Phychology, 11, 318-335.

Duda, J.L., M.T. Allison, 1990. Cross-cultural analysis in exerecise and sport psychology: a void in the field. Journal of Sport and Exercise Phychology, 12, 114-131. 
Duda, J.L., C. Hayashi, 1998. Measurement issues in cross-cultural research within sport and exercise psychology. In: Duda, J.L. (Ed.), Advances in sport and exercise psychology measurement (pp. 471-483). Morgantown, W.Va: Fitness Technology.

Fonseca, A., P.A. Brito, 2005. The issue of the cross-cultural adjustment of instruments for psychological evaluation in national sport contexts. The case of the Task and Ego Orientation in Sport Questionnaire (TEOSQ). Psychologica, 39, 95-118.

Guivernau, M., J.L. Duda, 1994. Psychometric properties of a Spanish version of the tast and ego orientation in sport questionnaire (TEOSQ) and beliefs about the causes of success inventory. Revista de Psicología del Deporte, 5, 31-51.

Hambleton, R. K. (2006). Adaptación de tests para su uso en diferentes idiomas y culturas: fuentes de error, posibles soluciones y directrices prácticas. In: Muñiz, J. (Ed.), Psicometría (pp. 207-238). Madrid, España: Universitas, S.A.

Helms, J.E., 1992. Why is there no study of cultural equivalence in standardized cognitive-ability testing. American Psychologist, 47, 1083-1101.

Hu, L., P.M. Bentler, 1995. Evaluating model fit. In: Hoyle, R. (Ed.), Structural equation modeling: Issues, concepts, and applications. Newbury Park, CA: Sage.

Hu, L., P.M. Bentler, 1999. Cutoff criteria for fit indexes in covariance structure analysis: Conventional criteria versus new alternatives. Structural Equation Modeling, 6(1), 1-55.

Kaiser, H.F., 1974. An index of factorial simplicity. Psychometrika, 39, 31-36.

Kim, B.J., L. Williams, D.L. Gill, 2003. A cross-cultural study of achievement orientation and intrinsic motivation in young USA and Korean athletes. International Journal of Sport Psychology, 34, 168-184.

Li, F., P. Harmer, L. Chi, N. Vongjaturapat, 1996. Cross-cultural validation of the task and ego orientation in sport questionnaire. Journal of Sport and Exercise Phychology, 18, 392-407.

López-Walle, J., I. Balaguer, J.L. Meliá, I. Castillo, J. Tristán, 2011. Adaptación a la población mexicana del Cuestionario de Orientación al Ego y a la Tarea en el Deporte (TEOSQ). Revista de Psicología del Deporte, 20(2), 523-536.

Marsh, H.W., K.T. Hau, Z. Wen, 2004. In search of golden rules: Comment on hypothesis-testing approaches to setting cutoff values for fit indexes and dangers in overgeneralizing hu and Bentler's (1999) findings. Structural Equation Modeling, 11(3), 320-341.

Moreno-Murcia, J.A., E. Huéscar, N. Parra, 2013. Manipulación del clima motivacional en Educación Física para evitar el aburrimiento. Revista Mexicana de Psicología, 30(2), 108-114.

Moreno-Murcia, J.A., C. Martínez-Galindo, V. Moreno, P.J. Marcos Pardo, L. Conte, R. Moreno González, 2012. Motivación, creencias de habilidad e intención de ser físicamente activo al finalizar la Educación obligatoria. Revista Mexicana de Psicología, 29(2), 175-183.

Muñiz, J., P. Elosua, R.K. Hambleton, 2013. Directrices para la traducción y adaptación de los tests: segunda edición. Psicothema, 25(2), 151-157.

Nicholls, J. G. (1989). The competitive ethos and democratic. Cambridge, MA: Harvard University Press.

Papaioannou, A., O. Kouli, 1999. The effect of task structure, perceived motivational climate and goal orientations on students' task involvement and anxiety. Journal of Applied Sport Psychology, 11(1), 51-71.

Papaioannou, A., A.I. Macdonald, 1993. Goal perspectives and purposes of physical education as perceived by greek adolescents. Physical Education Review, 16(1), 41-48.

Peiró, C., J.R. Sanchis, 2004. Las propiedades psicométricas de la versión inicial del cuestionario de orientación a la tarea y al ego (TEOSQ) adaptado a la educación física en su traducción al castellano. Revista de Psicología del Deporte, 13(1), 25-39.

Raykov, T., G.A. Marcoulides, 2006. A first course in structural equation modeling. Mahwah, NJ: Erlbaum. 
Stuntz, C.P., M.R. Weiss, 2012. Achievement goal orientations and motivational outcomes in youth sport: The role of social orientations. Psychology of Sport \& Exercise, 10, 255-262.

Todorovich, J.R., M.D. Curtner-Smith, 2002. Influence of the motivational climate in physical education on sixth grade pupils' goal orientations. European Physical Education Review, 8(2), 119-138.

Van-Yperen, N.W., J.L. Duda, 1999. Goal orientations, beliefs about success, and performance improvement among young elite Dutch soccer players. Scandinavian Journal of Educational Research, 9, 358-364.

Wakayama, H., E. Watanabe, K. Inomata, 2002. Exploratory factor analysis of the Sport Orientation Questionnaire and the Task and Ego Orientation in Sport Questionnaire in a Japanese sport setting. Perceptual \& Motor Skills, 95, 1179-1186.

Walling, M.D., J.L. Duda, 1995. Goals and their associations with beliefs about success in and perceptions of the purposes of physical education. Journal of Teaching in Physical Education, $14(2), 140-156$. 